\title{
Agitación psicomotriz e implicaciones médico forenses
}

\author{
Psychomotor agitation and forensic implications
}

\section{Resumen}

Observaciones llevadas a cabo durante los últimos 15 años por expertos forenses han dado lugar al reconocimiento de un nuevo síndrome que puede llevar a la muerte súbita e inesperada. La muerte por agitación psicomotriz generalmente se acompaña por un procedimiento de restricción llevado a cabo por fuerzas y cuerpos de seguridad del estado, cuerpos de seguridad privada o personal hospitalario. Ello conlleva que dicho fallecimiento sea catalogado dentro del grupo de muertes en privación de libertad, muertes en custodia o deaths in custody. La frecuencia de este cuadro, Ilamativo por las circunstancias extrañas en las que se produce, es cada vez mayor ya que uno de sus principales factores de riesgo como es el consumo de drogas, fundamentalmente la cocaína, aumenta de forma alarmante en la sociedad de nuestros días.

Por tanto, partiendo de la hipótesis de que la agitación psicomotriz de origen tóxico o psiquiátrico puede acabar en la muerte del sujeto tras una retención prolongada así como que algunos de los sujetos que sufren un cuadro de agitación psicomotriz son ingresados en el hospital durante o tras dicho episodio, nuestro objetivo ha sido realizar un estudio descriptivo utilizando para ello una muestra de 31 casos que requirieron atención médica en el Hospital de Poniente entre los años 2003 y 2005. Esta muestra la dividimos en dos subgrupos:

- Sujetos con agitación psicomotriz y análisis cualitativo de tóxicos positivo y,

- Sujetos con agitación psicomotriz y análisis cualitativo de tóxicos negativo.

Con esta división en dos grupos realizamos un estudio comparativo entre ambos analizando estadísticamente diferentes variables con el fin de determinar la influencia de las drogas psicoactivas en los cuadros de agitación psicomotriz.

Los resultados obtenidos ponen de manifiesto la falta de datos en las historias clínicas, el escaso número de estudios relacionados con el tema en cuestión y la ausencia de diferencias estadísticamente significativas entre los pacientes con agitación psicomotriz y consumo de tóxicos de los que no han consumido recientemente.

Palabras clave: Agitación psicomotriz. Cocaína. Delirium agitado. Muertes en custodia.

\section{Abstract}

Observations carried out by forensic experts over the last 15 years lead us to the recognition of a new syndrome that can take to a sudden and unexpected death. Death caused by psychomotor agitation is generally accompanied by a restriction procedure carried out by security forces, private security or hospital staff. It entails that this death can be classified within the group of deaths in deprivation of liberty or deaths in custody. The frequency of this condition, remarkable for the strange circumstances in which it occurs, is growing as one of their major risk factors such as drugs, primarily cocaine, increases dramatically in today's society.

Therefore, based on the hypothesis that psychomotor agitation due to toxic or psychiatric origin can end in the death of the subject after a prolonged retention as well as some of the subjects suffering from psychomotor agitation are admitted to hospital during or after this episode, our goal was to perform a descriptive study using a sample of 31 cases requiring medical attention in the Poniente Hospital between 2003 and 2005. This sample was divided into two subgroups:

\section{F. Martín Cazorla ${ }^{1}$ IM. Santos Amaya² M. López García ${ }^{3}$}

${ }^{1}$ Médico Forense. Servicio de Patología Forense del Instituto de Medicina Legal de Málaga.

Especialista en

Medicina Legal y

Forense.

Profesor Titular del Área de Medicina Legal y Forense de la Facultad de Medicina de la Universidad de Málaga.

${ }^{3}$ Especialista en Medicina Familiar y Comunitaria.

Correspondencia: Dr. Fernando Martín C/ Fiscal Luis Portero; Ciudad de la Justicia IML Málaga,

Servicio de Patología Forense.

E-mail:

fermarcaz@hotmail.com

Fecha de recepción: 9.MAR.2011

Fecha de aceptación:

13.JUN.2011 
- Patients with psychomotor agitation and positive toxic qualitative analysis and,

- Patients with psychomotor agitation and negative toxic qualitative analysis.

A comparative study was carried out between the two different groups, statistically analyzing different variables to determine the influence of psychoactive drugs in the state of psychomotor agitation.

Results highlight the lack of data in medical records, the small numbers of studies related to the topic and no statistically significant differences between patients with psychomotor agitation and toxic consumption of those who have not recently consumed.

Key words: Psychomotor agitation. Cocaine. Excited delirium. Deaths in custody.

\section{Introducción}

La agitación psicomotriz constituye un reto para los profesionales sanitarios, ya que habitualmente desbordan sus habilidades de manejo. Esta situación representa una de las casuísticas más prevalentes en las urgencias hospitalarias siendo por ello necesario que los profesionales conozcan los aspectos básicos de la intervención con la finalidad de poder evitar la escalada que acontece en no pocas situaciones. También es de capital importancia conocer las diferentes etiologías subyacentes, así como los principios elementales para el manejo farmacológico de estos casos.

Aunque la agitación es bastante común en el ámbito hospitalario, sólo dos estudios han evaluado su incidencia y factores de riesgo. Ya Fraser et a/ ${ }^{1}$ investigaron el efecto de la edad sobre la incidencia de la agitación psicomotriz, y Woods et al ${ }^{2}$ estudiaron la incidencia, factores de riesgo y resultados de la agitación en la $\mathrm{UCl}$ con pacientes que requerían ventilación mecánica.

La mayoría de investigaciones que han estudiado las enfermedades neuropsiquiátricas no se han centrado en la agitación pero han evaluado el delirium o el síndrome de UCl usando diferentes definiciones y criterios diagnósticos.

Existen pocos estudios descriptivos y comparativos que abarquen a la agitación psicomotriz y sobre todo a aquellos que tienen a la enfermedad mental o al consumo de tóxicos en su origen. Cada año un pequeño número de personas fallecen de forma súbita mientras son retenidos. Muchas de estas muertes se asocian con individuos que fueron retenidos por las fuerzas de seguridad; otras muertes acontecían durante la retención en personas que están bajo la influencia de medicación psiquiátrica. Hay una gran confusión acerca de las causas y circunstancias que rodean a estas muertes súbitas durante la restricción. Es difícil conocer la causa de estas muertes, de hecho los médicos tienen una enorme dificultad en identificar la causa de la muerte sólo a través de la autopsia.

\section{Objetivos}

Partiendo de la hipótesis de que la agitación psicomotriz de origen tóxico o psiquiátrico puede acabar en la muerte del sujeto tras una retención prolongada así como que algunos de los sujetos que sufren un cuadro de agitación psicomotriz son ingresados en el hospital durante o tras dicho episodio, uno de nuestros objetivos ha sido realizar un estudio descriptivo utilizando para ello una muestra de 31 casos que requirieron atención médica en el Hospital de Poniente (El Ejido, Almería) entre los años 2003 y 2005 . Esta muestra la dividimos en dos subgrupos:

- Sujetos con agitación psicomotriz y análisis cualitativo de tóxicos positivo.

- Sujetos con agitación psicomotriz y análisis cualitativo de tóxicos negativo.

Con esta división en dos grupos posteriormente realizamos un estudio comparativo entre ambos analizando estadísticamente diferentes variables con el fin de determinar la influencia de las drogas psicoactivas en los cuadros de agitación psicomotriz.

Debido al desconocimiento generalizado de dicho cuadro a nivel médico, social y de personal de seguridad, se pretende a través de este estudio aportar un mayor conocimiento de los cuadros de agitación psicomotriz de origen tóxico así como sus diferencias con los de origen no tóxico para:

- Formación, fundamentalmente a nivel médico y personal de seguridad.

- Prevención de las posibles muertes en casos de agitación de origen tóxico.

- Divulgación a diferentes niveles. 


\section{Material y métodos}

El estudio fue realizado en el Hospital de Poniente que atiende a una población de 225.000 habitantes. Una vez obtenido el permiso por parte de la Comisión de Docencia e Investigación de la Empresa Pública Hospital de Poniente, realizamos una revisión de todos los historiales clínicos de los enfermos estudiados e ingresados en el Hospital de Poniente con el diagnóstico de agitación psicomotriz entre enero de 2003 y diciembre de 2005.

Finalmente se han seleccionado los pacientes tratados en el área de urgencias o ingresados en la Unidad de Salud Mental, descartando aquellos otros que presentaban una enfermedad orgánica subyacente como etiología del cuadro de agitación. Como consecuencia de todo ello fueron incluidos para el estudio un total de 31 pacientes.

Algunas variables han sido desestimadas por ser poco específicas en relación con nuestro objeto de estudio o por encontrarse registradas en un escaso número de historias clínicas. Por otra parte, de los tóxicos detectados no se pudieron tener los datos cuantitativos al no realizarse este tipo de estudio de forma protocolizada en el hospital.

Las variables utilizadas se describen en la Tabla 1. La ficha se trasladó a una base de datos realizada con el programa Excel (Microsoft Office 2003). La estadística descriptiva se realizó con el programa SPSS para Windows, versión 13.0., de SPSS Inc.

\section{Resultados}

Los resultados obtenidos han sido agrupados en dos grandes categorías:

1. Resultados generales.

2. Resultados obtenidos de la comparación de ambos grupos.

\section{Resultados generales}

Del total de la muestra (31 pacientes), 28 eran varones y 3 fueron mujeres; la edad media fue de 33,35 años, con un rango de edad que osciló entre los 19-60 años. Estos pacientes constituyen el $0,029 \%$ de los usuarios que acuden a urgencias.

Con respecto al estado civil de los pacientes, 13 estaban solteros, 8 casados ( 1 de ellos era mujer), 8 separados (1 de ellos era mujer) y 1 divorciado.

Durante los años de nuestro estudio (2003-2005), la época del mismo en la que sucedió el episodio de agitación psicomotriz fueron: 13 en invierno, 10 en primavera, 5 en verano y 3 en otoño.

Respecto al estudio descriptivo obtuvimos los siguientes resultados:

a) La agitación psicomotriz tiene un predominio mayoritario en el sexo masculino (9/1) con una edad media de 33,35 años.

\begin{tabular}{|c|c|c|c|c|c|}
\hline $\begin{array}{l}\text { Variables } \\
\text { epidemiológicas }\end{array}$ & $\begin{array}{c}\text { Datos de llegada } \\
\text { a urgencias }\end{array}$ & $\begin{array}{l}\text { Datos referentes } \\
\text { historia clínica }\end{array}$ & $\begin{array}{c}\text { Datos } \\
\text { analiticos }\end{array}$ & $\begin{array}{c}\text { Unidad de Salud } \\
\text { Mental (USM) }\end{array}$ & $\begin{array}{c}\text { Medidas } \\
\text { de contención }\end{array}$ \\
\hline Edad & Hora & $\begin{array}{l}\text { Antecedentes } \\
\text { psiquiátricos }\end{array}$ & $\begin{array}{c}\text { Peso/altura } \\
\text { Temperatura } \\
\text { Presión arterial } \\
\text { FC/FR }\end{array}$ & Diagnóstico CIE X & $\begin{array}{l}\text { Modalidad de } \\
\text { internamiento }\end{array}$ \\
\hline Sexo & $\begin{array}{l}\text { Persona que lo } \\
\text { traslada }\end{array}$ & $\begin{array}{l}\text { Ingresos previos } \\
\text { en USM }\end{array}$ & Glucemia & Días de estancia & $\begin{array}{l}\text { Restricción } \\
\text { empleada }\end{array}$ \\
\hline Estado civil & $\begin{array}{l}\text { Tiempo en } \\
\text { urgencias }\end{array}$ & $\begin{array}{c}\text { Análisis } \\
\text { cualitativo tóxicos }\end{array}$ & Saturación & Derivación al alta & $\begin{array}{l}\text { Tipo de } \\
\text { restricción física }\end{array}$ \\
\hline Profesión & $\begin{array}{l}\text { Derivación desde } \\
\text { urgencias }\end{array}$ & & $\mathrm{pH}$ & & $\begin{array}{l}\text { Tipo de } \\
\text { restricción } \\
\text { química }\end{array}$ \\
\hline
\end{tabular}

Tabla 1.

Variables analizadas. 
b) El antecedente psiquiátrico más frecuente fue el trastorno psicótico seguido de la toxicomanía (14/9)

c) Todos los pacientes excepto uno fueron ingresados en la Unidad de Salud Mental; la modalidad de internamiento voluntaria fue más frecuente que la involuntaria (18/10).

d) Mayoritariamente (28) los pacientes con agitación psicomotriz requirieron algún tipo de restricción. En todos los casos se utilizó la contención química ya sea de forma aislada o asociada a la física; esta última nunca se empleó de forma aislada. Dentro de la contención química o farmacológica se utilizó fundamentalmente la combinación de neurolépticos y ansiolíticos. Los datos en relación con la restricción física eran escasos en los informes de la Unidad de Salud Mental.

e) La mayor parte de los pacientes habían estado internados previamente en el servicio de psiquiatría, fundamentalmente los pacientes con agitación psicomotriz y análisis cualitativo a drogas en orina negativo (grupo 0 ).

Tabla 2.

Tipo de tóxicos.

\begin{tabular}{|l|c|}
\hline Tóxico & $N^{0}$ pacientes \\
\hline Cocaína & 6 \\
\hline Opiáceos & 4 \\
\hline Cannabis & 8 \\
\hline Benzodiacepinas & 14 \\
\hline Metadona & 1 \\
\hline Anfetaminas & 1 \\
\hline Analgésicos no opioides & 1 \\
\hline
\end{tabular}

Tabla 3. Análisis comparativo de ambos grupos.

\begin{tabular}{|c|c|c|}
\hline VARIABLES & GRUPO 0 & GRUPO 1 \\
\hline Edad & $39(11,25)^{*}$ & $29(10,03)$ \\
\hline $\mathrm{T}^{\circ}$ espera & $475(414,42)$ & $268(219,99)$ \\
\hline Temperatura & $36,8(0,81)$ & $36,7(0,45)$ \\
\hline Presión arterial & $131-80(15,56)$ & $128-76(16,10)$ \\
\hline Frecuencia cardiaca & $89,5(24,67)$ & $90,27(15,16)$ \\
\hline Glucemia & $195(71,62)$ & $127(67,32)$ \\
\hline Saturación & $96(3,78)$ & $93(5,65)$ \\
\hline $\mathrm{pH}$ & - & $7,1(0,31)$ \\
\hline Antecedentes psiquiátricos & $81,8 \%$ & $100 \%$ \\
\hline Internamiento involuntario & $42 \%$ & $31 \%$ \\
\hline Contención mixta & $75 \%$ & $43 \%$ \\
\hline Internamientos previos & $83,3 \%$ & $46,7 \%$ \\
\hline $\begin{array}{l}\text { Derivación al alta } \\
{ }^{*} \text { (Desviación típica) }\end{array}$ & ESM distrito & ESM distrito \\
\hline
\end{tabular}

f) La estancia media en la Unidad de salud Mental fue de 12,48 días (la desviación típica fue de 17,99$)$.

g) Una vez dados de alta, en el informe constaba el diagnóstico psiquiátrico que ocasionó el cuadro de agitación psicomotriz. El cuadro predominante fue el trastorno mental 0 del comportamiento debido al consumo perjudicial de drogas o sustancias psicotropas ( 3 debido a alcohol, 2 a cannabinoides, 2 a cocaína y 9 a múltiples drogas), seguido del trastorno bipolar (5) y de la esquizofrenia (3).

h) El seguimiento de los pacientes dados de alta hospitalaria lo hacía fundamentalmente el equipo de salud mental de distrito.

i) Los tóxicos más detectados entre los pacientes con agitación psicomotriz y análisis cualitativo a drogas en orina positivo (grupo 1) fueron benzodiacepinas (14), cannabis (8) y cocaína (6) (Tabla 2)

\section{Resultados obtenidos de la comparación de ambos grupos}

Los 31 pacientes incluidos en nuestro estudio fueron clasificados en dos grupos en función del resultado cualitativo a tóxicos en el momento del ingreso:

- Grupo 0: pacientes cuyo resultado analítico a drogas en el momento del ingreso fue negativo; el número total de pacientes fue de 9 .

- Grupo 1: pacientes cuyo resultado analítico a drogas en el momento del ingreso fue positivo; el número total de pacientes fue de 17.

En 5 pacientes no constaba en su historial clínico ningún dato analítico relativo al consumo de tóxicos.

En la Tabla 3 podemos observar el análisis diferencial entre ambos grupos.

\section{Discusión}

En nuestro estudio tenemos una muestra constituida por 31 casos de pacientes que a su llegada a urgencias presentan o han presentado una crisis de agitación psicomotriz en sus diversas variantes, desde una inquietud psicomotora hasta episodios de agresión verbal y/o física. Las historias clínicas estudiadas eran incompletas en cuanto a los datos de dicha crisis de agitación psicomotriz. En la hoja de urgencias no siempre se recogían datos como las constan- 
tes vitales y los episodios de agitación psicomotriz no eran definidos de forma exhaustiva por el médico de urgencias; esto sólo ocurría cuando eran explorados por el psiquiatra. La muestra estudiada es acorde con los datos recogidos en otros estudios, como los 33 casos de Tintinalli y Mc $\mathrm{Coy}^{3}$ o los 16 casos del estudio de Nashville ${ }^{4}$, aunque es sensiblemente menor al número de pacientes estudiados por el Dr. Samir Jaber ${ }^{5}$ (219 pacientes). En este último caso, 29 pacientes fueron excluidos del estudio porque su patología no permitía una evaluación del nivel de conciencia por lo que la muestra se redujo finalmente a 182 pacientes.

La edad media de nuestros pacientes fue de 33,35 años, con un rango de edad que osciló entre los 19 y los 60 años. Si procedemos al análisis de los grupos en función del consumo de algún tipo de tóxico previo, podemos observar cómo la edad media del grupo consumidor (grupo 1) es sensiblemente menor a la del grupo no consumidor (grupo 0), estamos hablando de una edad media de 29,41 años frente a 39,5 años respectivamente. En el estudio de Nashville, la edad media de los pacientes violentos fue menor (32,5 $\pm 8,1$ años) que la de los pacientes que no eran violentos (44,3 \pm 23,9 años); estos datos vienen a confirmar los obtenidos con los pacientes agitados que llegaron a urgencias del Hospital de Poniente, los cuales tenían una media de edad muy próxima a los del estudio de Nashville (33,35 años de nuestro estudio frente a los 32,5 de NashviIle). En el estudio prospectivo de la agitación psicomotriz dirigido por el Dr. Samir Jaber del Hospital Universitario Saint Eloi de Montpellier entre los años 1999 y 2000, la edad media de los pacientes incluidos en dicho estudio fue de $60 \pm 15$ años; los resultados de este estudio son sensiblemente mayores a los obtenidos en el nuestro y ello puede ser debido a que en el estudio realizado por el Dr. Samir Jaber incluía a pacientes admitidos en la Unidad de Cuidados Intensivos por una patología médica (25\%) o quirúrgica $(75 \%)^{6}$. Hay que tener presente que en nuestro estudio se rechazaron aquellos casos cuyo cuadro de agitación psicomotriz presentaba como etiología una enfermedad orgánica (tumores, postoperatorios, etc.) así como los casos de delirium tremens.

Con respecto al sexo de los pacientes afectados por la agitación, en nuestro estudio, el 90,33\% fueron hombres y el $9,67 \%$ mujeres. Estos resultados vienen a corroborar los estudios de pacientes agitados cuya mayoría suelen ser hombres (65\% en el estudio del Dr. Samir Jaber) ${ }^{5,7}$.

En cuanto al consumo de tóxicos previo al cuadro de agitación psicomotriz podemos decir que en nuestro estudio, la muestra se vio reducida en 5 pacientes $(n=26)$ pues en la historia clínica no se hacía mención alguna a un consumo previo de tóxicos (ni en antecedentes personales ni dato analítico). En los restantes casos se puede observar como hay un predominio de los pacientes que han consumido al menos una droga antes del episodio (17 del grupo 1 frente a 9 del grupo 0); a su vez dentro del grupo 1 , el consumo fue de una única droga en el 35,29\% (en 5 casos benzodiacepinas y en un caso cannabis) y fue un policonsumo en el $64,70 \%$ destacando la combinación de cocaína y cannabis en 2 casos; cocaína, cannabis, benzodiacepinas y opiáceos en 2 casos; y finalmente en otros dos casos el consumo mixto de cannabis y benzodiacepinas.

Las drogas más consumidas fueron por orden: benzodiacepinas, cannabis, cocaína, opiáceos, y finalmente anfetaminas, metadona y analgésicos de forma similar. Sin embargo, debemos tener en consideración que algunos de nuestros pacientes podrían haber recibido benzodiacepinas como tratamiento en el lugar de la escena, puesto que en algunos casos fueron transportados al Servicio de Urgencias hospitalaria por personal médico (dispositivo del 061 o DCCU del centro de salud más cercano).

En el estudio del Dr. Samir Jaber, existía una historia previa de consumo de alcohol en el $30 \%$ de los casos y el $21 \%$ usó drogas psicoactivas antes de la hospitalización. En el estudio de Nashville, aunque no hay datos numéricos reflejados, habla de una proporción mayor del consumo de alcohol o drogas entre los pacientes más jóvenes que a su vez es el grupo de pacientes más violento ${ }^{8,9}$.

Uno de los estudios referentes a nivel mundial en relación con la agitación psicomotriz es el Epidemiological Catchment Area (Estudio Epidemiológico de Distrito $)^{10}$ donde 20.000 personas fueron entrevistadas en diversas poblaciones de Estados Unidos. En aproximadamente la mitad de la muestra, la información obtenida fue referente a comportamientos violentos. Estos informes revelaron que las probabilidades de comportamientos violentos en hombres y mujeres esquizofrénicas eran, respectivamente, 5,3 y 5,9 veces mayor que en personas sin diagnóstico de enfermedad mental. Este riesgo se incrementaba a 12,6 y 9,1 respectivamente cuando la enfermedad mental se asociaba al consumo de tóxicos.

En otro estudio ${ }^{11}$ realizado sobre la población norteamericana se examinó a 385 pacientes y 365 controles. El hecho de golpear a alguien, pelear y el uso de un arma fueron, respectivamente, 2,4 veces, 1,7 veces y 3,6 veces más frecuente en pacientes (de los cuales el 19,4\% eran esquizofrénicos) que en pacientes no esquizofrénicos. 
Casi el 50\% de los individuos con un diagnóstico establecido de esquizofrenia presenta también los criterios de dependencia y abuso de sustancias. En un estudio realizado en Estados Unidos se utilizaron los informes realizados por la policía y los hospitales, a partir de los cuales se obtuvo una muestra de 1.136 pacientes y 519 casos control. En dicho estudio ${ }^{12}$ no se hallaron diferencias significativas entre la prevalencia de la violencia en pacientes sin síntomas de consumo de tóxicos $(4,3 \%)$ y los controles también libres de síntomas de consumo de tóxicos (3,3\%). La existencia de síntomas de consumo de tóxicos incrementó significativamente la violencia a $14,1 \%$ de los pacientes, y a $11,1 \%$ de los controles.

Según L. Citrome et a/13,14 el comportamiento agresivo no es infrecuente en las unidades hospitalarias. Aproximadamente el $13 \%$ de un total de 253 pacientes atacaron físicamente a otra persona durante las primeras 24 horas en la unidad de salud mental. Los pacientes maníacos fueron los más propensos a ser agresivos con un $26 \%$ de los pacientes diagnosticados de trastorno bipolar (46 pacientes). En con-

Figura 1 Antecedentes psiquiátricos.

\section{Antecedentes psiquiátricos}

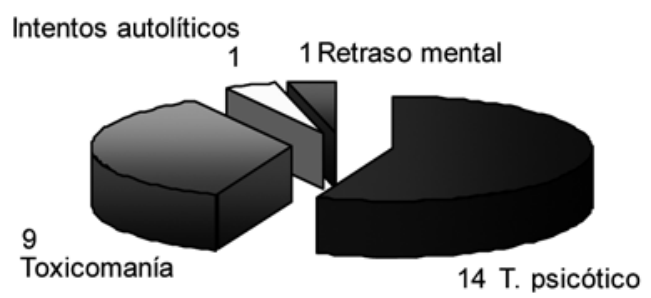

Figura 2. Llegada a urgencias.

\section{Llegada al Servicio de Urgencias}

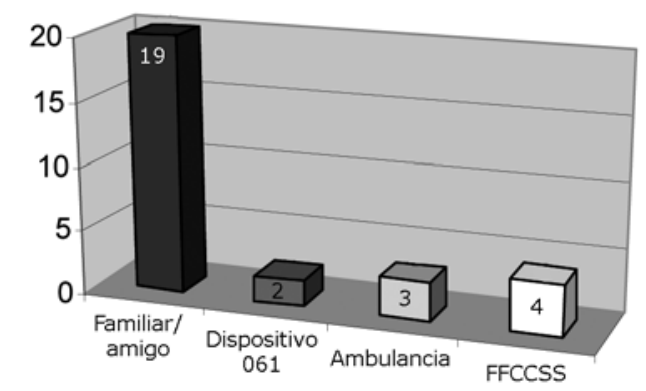

traste, aproximadamente el $10 \%$ de los 87 pacientes diagnosticados de esquizofrenia fueron agresivos durante ese mismo periodo de tiempo. Asimismo, un estudio $^{15}$ que observaba a los pacientes ingresados durante 8 días tras su admisión encontró que aproximadamente el 9\% de 289 pacientes diagnosticados de esquizofrenia o trastorno esquizoafectivo agredían a alguien al menos una vez. En nuestro estudio el antecedente psiquiátrico recogido con mayor frecuencia fue el trastorno psicótico (sin especificar modalidad) seguido de la toxicomanía y el retraso mental; en un caso se recogió antecedentes de intentos autolíticos previos (Figura 1). En los resultados obtenidos sí hemos encontrado diferencia estadísticamente significativa ( $p$ de 0,008) entre el consumo de drogas y la existencia de antecedentes psiquiátricos (entendido éste como la existencia de consulta previa en la Unidad de Salud Mental), algunas veces filiada y otras no. Estos resultados son acordes con los múltiples estudios que relacionan las drogas con la enfermedad mental; asimismo coincide con los estudios como el reseñado anteriormente en donde los pacientes con enfermedad psiquiátrica son más propensos a los cuadros de agitación psicomotriz y sus variedades (agresividad, delirium agitado).

\section{Conclusiones}

Del estudio realizado podemos enumerar las siguientes conclusiones:

\section{Respecto al estudio descriptivo podemos concluir:}

a) El perfil del paciente que presenta un cuadro de agitación psicomotriz es el de un varón de 33 años con antecedentes psiquiátricos que requiere un ingreso voluntario en la unidad de salud mental.

b) Los tóxicos más detectados en los pacientes del grupo 1 fueron las benzodiacepinas, cannabis y cocaína.

2. Respecto al estudio comparativo entre los pacientes con agitación psicomotriz y el análisis cualitativo a drogas en orina negativo (grupo 0) y positivo (grupo 1) hemos obtenido los siguientes resultados:

a) El antecedente psiquiátrico mayoritario entre los pacientes del grupo 0 fue el trastorno psicótico, mientras que en el grupo 1 fue la toxicomanía; en este apartado sí se obtuvo significación estadística.

b) Destaca la escasa intervención tanto del personal sanitario como de las Fuerzas y Cuerpos de 
Seguridad del Estado (FFCCSE) en el traslado del paciente agitado al hospital (Figura 2).

3. Durante la realización del estudio se puso de manifiesto la falta de cumplimentación de las historias clínicas, fundamentalmente en el servicio de urgencias a diferencia de los informes elaborados por el servicio de psiquiatría.
4. Existe una marcada ausencia de estudios en relación con la agitación psicomotriz.

5. Por lo tanto, en base a los resultados obtenidos en este estudio, no se observan diferencias en los cuadros de agitación psicomotriz en los que ha existido un consumo reciente de tóxicos de los que no ha existido dicho consumo previamente.

\section{Bibliografía}

1. Fraser G, Prato B, Riker R, et al. Frequency, severity, and treatment of agitation in young versus elderly patients in the ICU. Pharmacotherapy. 2000; 20: 75-82.

2. Woods J, Mion L, Connor J, et al. Severe agitation among ventilated medical intensive care unit patients: frequency, characteristics and outcomes. Intensive Care Med. 2004;30:1066-72.

3. Tintinally JE, Mc Coy M. Violent patients and the prehospital provider. Ann Emerg Med. 1993;22:1276-9.

4. Mock EF, Wrenn KD, Wright SW, Eustis TC, Slovis $\mathrm{CM}$. Prospective field study of violence in emergency medical services calls. Ann Emerg Med. 1998;32:33-6

5. Jaber S, Chanques G, Altairac C, et al. A prospective study of agitation in a medical-surgical ICU: incidence, risk factors, and outcomes. Chest. 2005;128(4):2749-57.

6. Woods J, Mion L, Connor J, et al. Severe agitation among ventilated medical intensive care unit patients: frequency, characteristics and outcomes. Intensive Care Med. 2004;30:1066-72.

7. Ely E, Stephens R, Jackson J, et al. Current opinions regarding the importance, diagnosis, and management of delirium in the intensive care unit: a survey of 912 healthcare professionals. Crit Care Med. 2004;32:106-12.

8. Brice JH, Pirrallo RG, Racht E, Zachariah BS, Krohmer J. Management of the violent patient. Prehosp Emerg Care. 2003;7:48-55.

9. Currier GW, Trenton A. Pharmacological treatment of psychotic agitation. CNS Drugs. 2002;16:219-28.

10. Regier D, Farmer M, Rae D, et al. Comorbidity of mental disorders with alcohol and other drug abuse. Results from the Epidemiologic Catchment Area (ECA) Study. JAMA. 1990;264:2511-8.

11. Link BG, Cullen FT, Andrews H. The violent and illegal behaviour of mental patients reconsidered. $A m$ Sociol Rev. 1992;57:275-92.

12. Dabrowski S, Frydman L, Zakowska-Dabrowska T. Physical restraint in Polish psychiatric facilities. Int J Law Psychiatry. 1986;8:369-82.

13. Citrome L. New treatments for agitation. Psychiatr Q. 2004;75(3):197-213.

14. Binder R, McNiel D. Effects of diagnosis and context on dangerousness. Am J of Psychiatry. 1988;145:728-32.

15. Tanke ED, Yesevage JA. Characterisitcs of assaultive patients who do and not provide visible cues of potential violence. Am J of Psychiatry. 1985; 142:1409-13. 